\title{
Research on Assembly Structure Design Method Based on BIM Technology
}

\author{
Guanlei Li ${ }^{1}$, Yadi Duan ${ }^{1}$ \\ ${ }^{1}$ Luohe Vocational Technology College, Henan, 462000, China
}

Keywords: BIM technology; fabricated structure; building resource library

\begin{abstract}
In recent years, the process of urbanization construction in China has been accelerating, the number of construction projects has been increasing and the scale of construction has been continuously expanding. While promoting China's economic and social development, there have also been some problems. In view of this, the application of advanced technologies in the construction field is becoming more and more. Among them, BIM technology is the commonly used technical means in construction. The construction personnel applied BIM technology to the process of assembly structure design, which further improved the overall quality of the design and improved work efficiency. Based on the above situation, this paper conducts relevant research on the assembly structure design based on BIM technology, hoping to provide valuable reference to relevant personnel and lay a good foundation for the construction and development of China's construction engineering.
\end{abstract}

\section{Structure design based on BIM model}

At this stage, the application of BIM technology in China's construction field has been very extensive. Many construction companies or construction units have incorporated BIM technology in the construction process of construction projects. For example, the design of the project, the management of the construction materials and the specific construction links. It can be said that BIM technology is an important technical means to ensure the sustainable development of the construction industry. In the process of assembly structure design, Chinese construction enterprises can apply BIM technology to various construction links, including production links, design links, construction links and management links and further realize the effective development of industrial construction projects $^{[1]}$.

In the process of assembling structure design, BIM technology should be used to establish the building resource design library. This link is an important link to ensure the smooth implementation of the assembly structure design. In the process of designing the building resource library, all resources related to building design should be integrated, including parts library, component library, civil building component library and electromechanical equipment library. In short, the building resource library is a relatively large resource library containing a lot of content, providing an effective reference for architectural design. At the same time, it is precisely because of the huge amount of data in the building resource pool that traditional management methods are difficult to meet the actual needs. With BIM technology, all relevant information can be managed efficiently and various parts and buildings can be managed hierarchically and in an orderly manner. Parts and components, these resources are uniformly recorded on the data form and a comprehensive table of material tables, doors and windows and other resources is produced accordingly, which facilitates effective management during construction. In addition, BIM technology can be used to decompose each construction link, produce appropriate parts according to the shape, size and quality required for each part of the decomposition, effectively promote production and construction and improve construction quality and efficiency ${ }^{[2]}$. The construction quantity of auxiliary construction in China using BIM system is shown in Figure 1. 


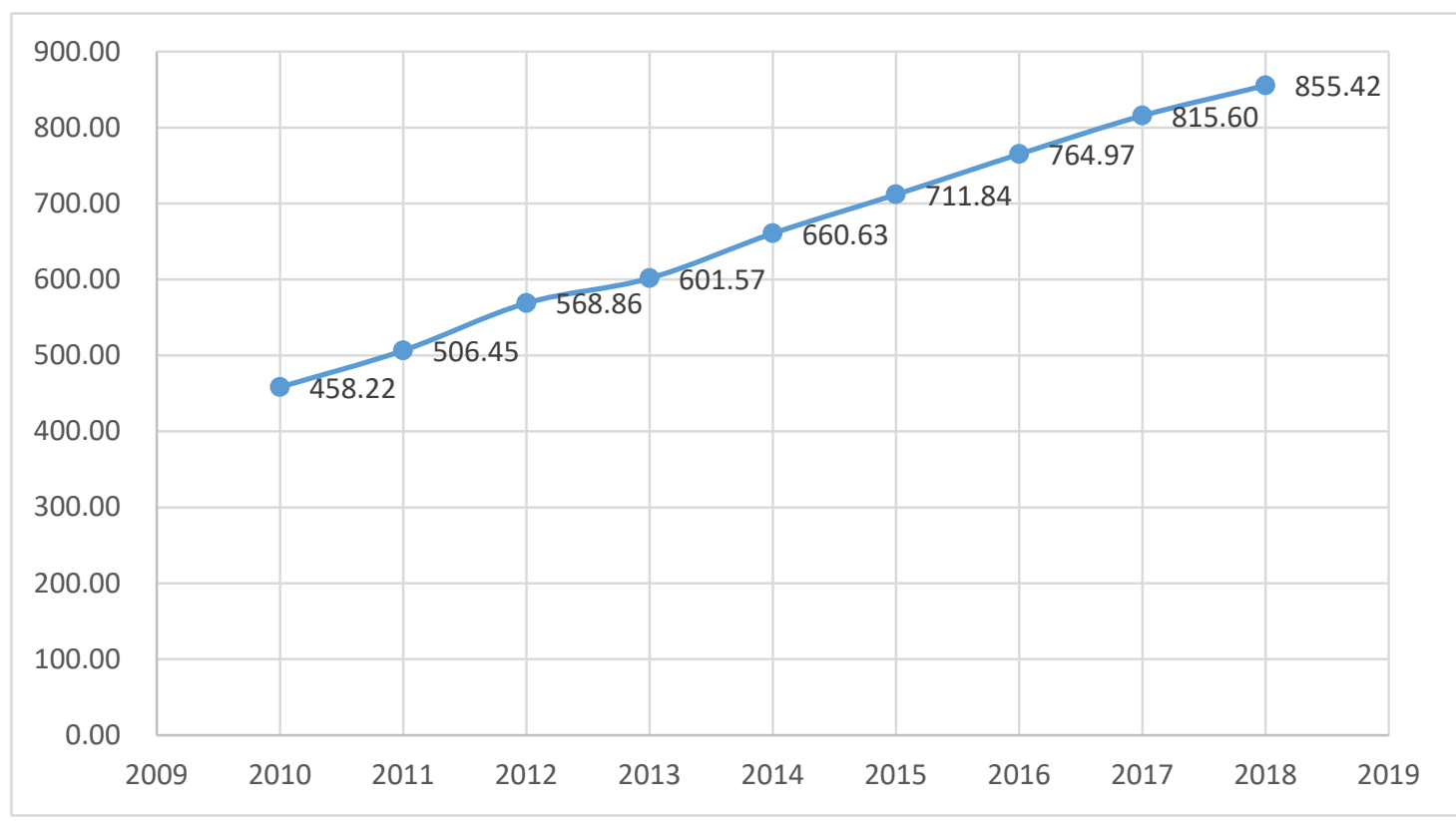

Figure 1 Construction quantity of auxiliary construction in China by BIM system

The traditional two-dimensional construction drawing and the BIM model established in Revit are very different, mainly in the following aspects. In comparison, it can be found that the information displayed by the traditional structural construction information map has great randomness. Similarly, this is the main reason for the failure to timely transmit structural information using traditional design software. At the same time, the use of traditional two-dimensional construction drawings is likely to lead to duplication of work, so the corresponding data can not be parameterized management. In fact, the Revit structural model can be regarded as an efficient parameterized information carrier and this data can be effectively transmitted to downstream software, thereby improving the efficiency of building design work cycle management. It can also be seen from the above discussion that BIM has an irreplaceable role in controlling construction progress and improving design quality. Compared with BIM design, the traditional two-dimensional structure diagram lacks relevant building height and direction information, which is also an important reason for its difficulty in spatial expression. For this reason, designers can only use their own imagination ${ }^{[3]}$. To restore the architectural design model, however, if some buildings with relatively complicated structures are restored by the imagination of the designer, it is inevitable that there will be some mistakes and omissions. If a certain degree of errors or omissions will seriously affect the quality of the buildings. If the platform is used for the construction work, because the design platform has the characteristics of color differentiation and $3 \mathrm{D}$ visualization, the related data can be visually managed by the relevant data, by trying to set the template and Adjustment can comprehensively check the model comprehensively, it is easier to find the problems in the design and greatly improve the quality of the drawing.

\section{Assembly structure design based on BIM data link technology}

The entire life cycle of a fabricated structure involves many steps, such as production, design and construction. With BIM technology, a large number of construction projects can be layered. For example, the building structure, plumbing engineering and other parts are professionally processed and the information and data of these links are integrated and shared. Under the three-dimensional and four-dimensional design concepts, the construction schemes of each link are displayed and the staff can clearly and intuitively Analyze the construction design plan. This method is different from the traditional two-dimensional design, which can further improve the design quality of the construction scheme and improve work efficiency. Based on BIM technology, a component resource library is established to integrate all the information in the construction project, including the design link, production link, material transportation link, assembly link and management link of 
the construction plan. This management method can effectively Avoid the mistakes that occur when people pass information between people and reduce the risk of data information loss or data information matching errors. In short, the assembly structure design process based on BIM technology has the characteristics of higher efficiency and better quality ${ }^{[4]}$. The entire life cycle of a fabricated structure consists of many steps, the weights of which are shown in Table 1.

Table 1 Weights of entire life cycle in a fabricated structure

\begin{tabular}{cccc}
\hline Index & Production & Design & Construction \\
\hline Weight & 0.372 & 0.403 & 0.225 \\
\hline
\end{tabular}

\section{Deep design of fabricated structure based on BIM technology}

\subsection{BIM component split design}

In the process of using the BIM technology for the assembly structure design, considering the design depth factor, the main construction considerations include the floor structure and the wall structure when designing the construction flow chart and form a complete individual. In the process of in-depth design, the above design links need to be split and the continuous models or components are separated one by one to form an independent part. A comprehensive analysis of these independent parts is carried out to calculate the part. The required number of components, quality standards and other aspects and then the production and processing of components. It should be noted that in the process of component splitting, it should be determined in accordance with the relevant standards set by the state and the production design requirements of the factory, to minimize the types of manufacturing components and to facilitate the mass production and processing of components in the factory. After formulating the splitting scheme of the components, the BIM technology can be directly used to build the three-dimensional component model and the original component design model can be split into different component connecting parts.

\subsection{BIM deepening the generation of drawings}

After establishing the 3D BIM component model, the staff needs to convert the various component data of the production and processing information into a two-dimensional design drawing and then use the drawing to complete the processing and production of the component. Generally speaking, the scale of construction projects is relatively large, the number of components required is also very large and the types of components are also various. The workload of traditional industrial prefabrication processes is very large and it is prone to calculation errors and inconsistent standards. The problem. With the application of BIM technology, the above problems are effectively solved. With the help of BIM technology, with the relevant computer software, automated component data analysis can be realized and related models of production components can be established ${ }^{[5]}$. Different from the traditional two-dimensional design scheme, the component design based on BIM technology can establish a three-dimensional model. When a certain index changes, the related work indicators or data information will automatically change, which reduces the design of the construction scheme. The problem of information does not match.

\section{Conclusion}

Through the discussion in this paper, it is shown that BIM technology plays an important role in the fabricated structure. Based on BIM technology, the staff can efficiently complete the splitting, design and industrial construction site management of the building model and effectively link all construction links together. Therefore, the research on the assembly structure design based on BIM technology will promote the architectural design concept, the innovative construction management concept and promote the transformation and development of the construction industry. It is believed that through the continuous deepening of research, the development level of China's construction industry will continue to improve, laying a good foundation for the construction and development 
of China's economy and society.

\section{References}

[1] Kyungki Kim,Yong Cho,Sijie Zhang. Integrating work sequences and temporary structures into safety planning: Automated scaffolding-related safety hazard identification and prevention in BIM[J]. Automation in Construction,2016,70.

[2] Heap-Yih Chong,Cen-Ying Lee,Xiangyu Wang. A mixed review of the adoption of Building Information Modelling (BIM) for sustainability[J]. Journal of Cleaner Production,2017,142.

[3] A.H. Oti,E. Kurul,F. Cheung,J.H.M. Tah. A framework for the utilization of Building Management System data in building information models for building design and operation[J]. Automation in Construction,2016,72.

[4] Guangbin Wang,Jiule Song. The relation of perceived benefits and organizational supports to user satisfaction with building information model (BIM)[J]. Computers in Human Behavior,2017,68.

[5] Zhao Xu,Yang Zhang,Xiayan Xu. 3D visualization for building information models based upon IFC and WebGL integration[J]. Multimedia Tools and Applications,2016,75(24). 